\title{
On Relevance of Linguistic Summaries - A Case Study from the Agro-Food Domain
}

\author{
Anna Wilbik ${ }^{1(\bowtie)}\left(\mathbb{0}\right.$, Diego Barreto $^{1}$, and Ge Backus ${ }^{2}$ \\ 1 Information Systems, Eindhoven University of Technology, \\ Eindhoven, The Netherlands \\ a.m.wilbik@tue.nl \\ 2 Connecting Agri and Food, Uden, The Netherlands \\ g.backus@connectingagriandfood.nl
}

\begin{abstract}
We present an application of linguistic summaries in the agro-food domain. We focus on the relevance aspect. Using the interviews we determine which linguistic summaries are useful and appropriate for target users (farmers). The user evaluation with a TAM survey indicates that linguistic summaries allow farmers to understand quickly the past performance of their pig barns.
\end{abstract}

Keywords: Linguistic summaries $\cdot$ Relevance $\cdot$ Case study Computing with words

\section{Introduction}

Recent developments in IoT technology allow more and more data to be collected. For instance, in agro-food domain sensor data can improve the crop yield and quality prediction [29,31] and optimization of food supply [34]. Those data can be analyzed through statistical analysis $[13,15]$, machine learning methods [12] or visual analytics [32,40]. Visual analytics technology is often used for the descriptive analytics purposes and understanding the data. However, many dashboards fail to communicate efficiently and effectively to the user [11]. Also, novice professionals struggles to understand simple graphs, especially if the information is not clear-cut and visually prominent [33]. Therefore, presenting the information in another way than visual, such as verbal, may be beneficial for the user.

One of the ways to obtain verbalization of data is to employ the linguistic summarization approach. Linguistic summaries can automatically generate natural language like sentences with aim of capturing the essence of data [16,24]. Linguistic summaries have proven to be useful in several applications, e.g. retail [17] and eldercare [39].

Linguistic summaries are being developed and matured as the method by many researchers, for instance by proposing new quality measures, e.g., [8,17], or more efficient generation methods $[10,18,28]$.

(C) Springer Nature Switzerland AG 2020

M.-J. Lesot et al. (Eds.): IPMU 2020, CCIS 1237, pp. 289-300, 2020.

https://doi.org/10.1007/978-3-030-50146-4_22 
Yet, one of the remaining challenges is that often too many linguistic summaries are being generated [10]. Thus, it is important to determine which linguistic summaries are useful and appropriate for target users achieving a balanced trade-off between complexity and precision of information [28]. Naturally usefulness or relevance is very much context dependent. In this paper we tackle this issue, by proposing a method to detect relevant linguistic summaries in a case from the agro-food domain. We consider a case of a pig barn and a climate sensor that captures the conditions in the barn. We want to present to the farmers only the linguistic summaries that they consider useful and allowing them to take actions that improve their operations.

This paper is structured as follows. Next section describes the background on linguistic summarization, Sect. 3 describes the context of the case study and Sect. 4 the proposed the method to detect relevant summaries. Section 5 presents the results of the case study and is followed by the concluding remarks.

\section{Background}

In this paper we follow the approach of Yager [41], which was considerably advanced and then implemented by Kacprzyk [16], Kacprzyk and Yager [21], Kacprzyk et al. [22,23].

In this approach linguistic data summaries are quantified propositions with two possible protoforms (or templates):

- simple protoform:

$$
Q y \text { 's are } P \text {; }
$$

e.g. Most cars are new

- extended protoform:

$$
Q R y \text { 's are } P
$$

e.g. Most new cars are fast

where $Q$ is the quantifier, $P$ is the summarizer, and $R$ is an optional qualifier, which are all modeled as fuzzy sets over appropriate domains.

The truth value, describing the validity of the summary, is the basic measure of the quality of the summary. Many methods for calculating the truth value have been proposed [8]. But the truth value is not the only quality measure of a linguistic summary. Kacprzyk et al. [17,23] proposed four additional measures, namely the degree of specificity, the degree of appropriateness, the degree of covering, and the length of the summary. Bugarin et al. [5] were differentiating between evaluating a single summary sentence and a set of summaries. They proposed several measures that capture aspects, such as coverage, length, and specificity. An overview of different quality criteria can be found in [8,28].

Those quality measures can be used to select a smaller set of the true summaries to be shown to the user. Three different approaches of employing the other quality measures can distinguished, namely using the thresholds [4,39], dominance of linguistic summaries [6] and aggregating the quality measures $[9,17]$. Yet, despite those methods, still too many linguistic summaries are obtained [10]. 
Linguistic summaries has been applied to different types of data: numerical $[7,14,36]$, time series $[19,20,30]$, sensor data [35,39], texts [37], videos [1-3] and processes $[10,38]$ in different application domains, such as retail [17], eldercare [39] or finance [19].

\section{Case Study in the Agro-Food Domain}

In this section we describe the context of the case study as the context is very important for the relevance. We consider a case of a climate sensor installed in a pig barn. This sensor measures the temperature, air humidity and carbon dioxide $\left(\mathrm{CO}_{2}\right)$ inside the barn every $10 \mathrm{~min}$. A dashboard was created to present the data collected by the sensor and some additional readings coming from the nearby weather station like outside air temperature and outside air humidity.

The dashboard initially showed the live gauges displaying the information about current values of inside temperature, humidity and $\mathrm{CO}_{2}$. Also three time series plots were introduced to display the data (temperature, humidity and $\mathrm{CO}_{2}$ ) over a longer period of time, typically 28 days. However, many farmers found this dashboard difficult to understand and deduce appropriate actions. To overcome this difficulty, it was decided to extend the dashboard with linguistic summaries.

Through discussions with the domain experts and a selected group of farmers (users) a few decisions were made. Firstly, it was decided to use only simple type protoforms, as they can provide the overview on the past. Moreover it was decided to create linguistic summaries about inside humidity, $\mathrm{CO}_{2}$, and daily range of inside temperatures (maximum-minimum), as the variations in the temperature in the barn is more important that the actual values. Thirdly, three linguistic labels for each variable and five quantifiers were defined and validated. Their membership functions are depicted in Figs. 1 and 2, respectively. The quantifiers has been designed in such a way, for a given argument only one of them has a value of membership function higher than 0.7 . This means that at most only one summary will be generated for a summarizer. By this, we will not present to the user both summaries as e.g. "Almost all $\mathrm{CO}_{2}$ levels are too high" and "Most $\mathrm{CO}_{2}$ levels are too high", as the second sentence is obsolete. Also each of the variables has desired values and undesired values. Temperature range and $\mathrm{CO}_{2}$ has undesired values only on one end, while humidity has undesired values on both ends. At last it was decided also to display at most two summaries per variable for comprehensibility purposes [27].

Also, the farmers indicated that they want to see information that triggers them for an action. Therefore linguistic summaries like "almost all $\mathrm{CO}_{2}$ values are OK" they don't find relevant and useful.

\section{Method for Selecting Relevant Linguistic Summaries}

The input from the domain experts and the farmers was used to propose a method that determined which of the true summaries can be considered as relevant and presented to the users. The most important feature of this case is 

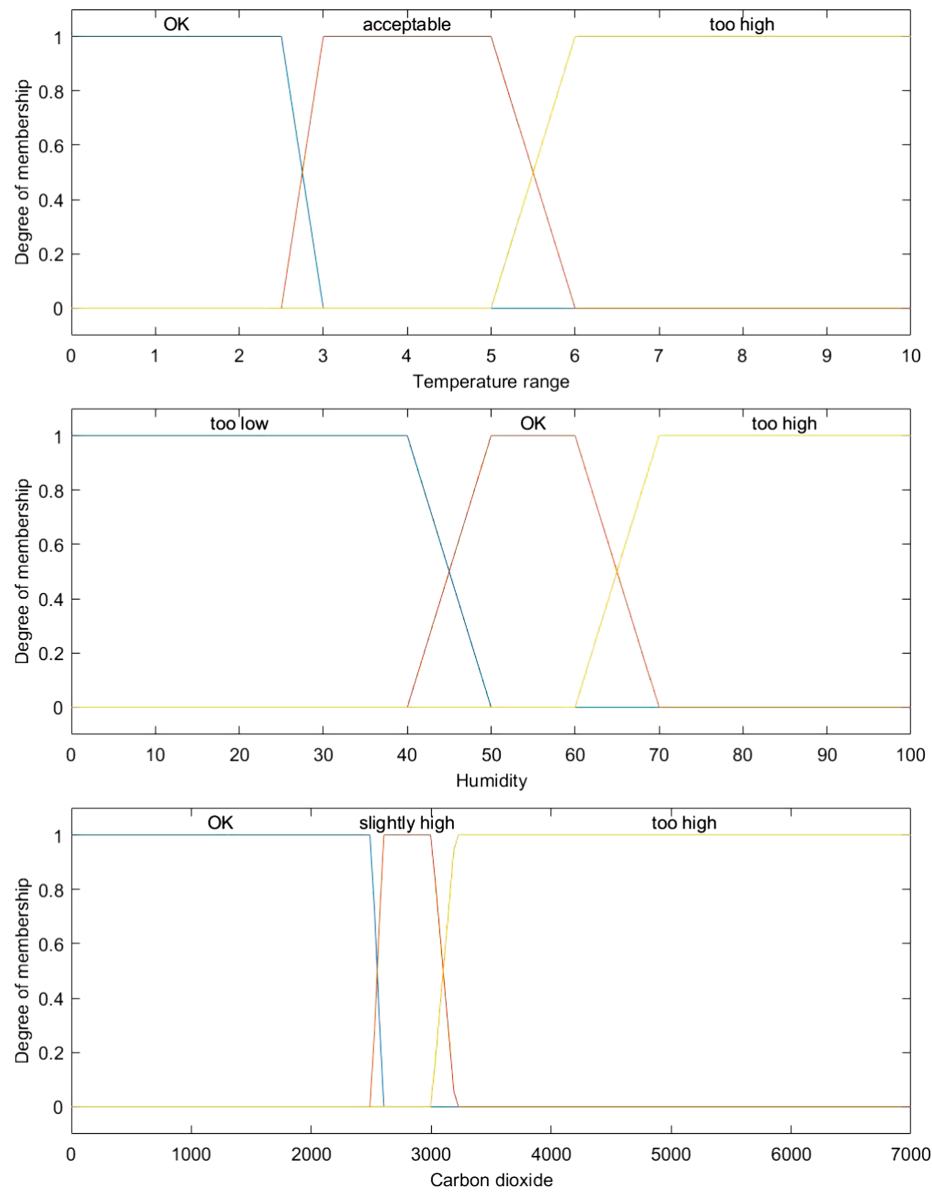

Fig. 1. Membership functions for the linguistic variables of temperature range, humidity and $\mathrm{CO}_{2}$.

that there is a desired state and users are only interested when things are not as expected. When considering those two requirements we can distinguish two cases:

- desired case - when most (if not all) data are in the desired (ok) range

- undesired case - when majority of the data is not in the desired (ok) range

In order to develop the method that selects relevant summaries, some additional information from the users is needed. One of them is to rank of the linguistic labels for each variables. For the one tailed variables, like $\mathrm{CO}_{2}$ levels, this is rather trivial task, the more undesired the value, the higher priority has the linguistic label. For the two tailed variables, like the humidity, where desired values are in the middle of the domain, setting the priority can be more challenging, as both extreme values can have same priorities or one of them is more 


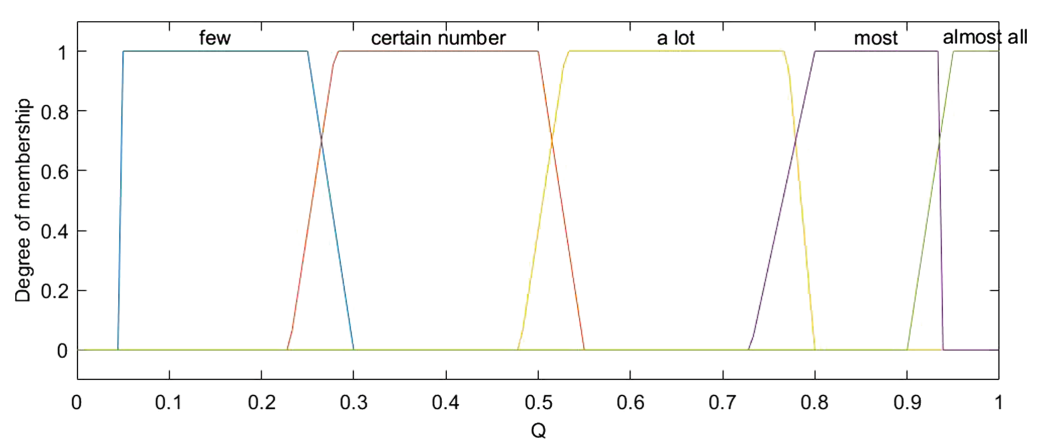

Fig. 2. Membership functions for quantifiers.

important than the other. We assume that priority of the qualifiers is positively correlated with the amount they describe, i.e. the bigger amount the qualifier describes, the higher the priority.

Second question is about what is more important to the user: summarizer or qualifier. For instance, consider the $\mathrm{CO}_{2}$ level. The question is which sentence is more useful to the user:

- certain number of the $\mathrm{CO}_{2}$ values is slightly high

- a few of the $\mathrm{CO}_{2}$ values is too high

In the considered case all farmers have indicated the second summary as more useful, giving the higher weight to the summarizer than qualifier.

The proposed method consist of 5 steps:

- Step 1: Calculate all summaries with truth value $\mathcal{T}>\theta_{T}$. We assumed $\theta_{T}$ of 0.7. For each linguistic value at most three summaries will be created.

- Step 2: Classify case as desired or undesired. This is done by looking at the summary with the biggest quantifier, in our case a lot, most or almost all. If the summarizer's label is a "desired" one, we consider a case as desired, otherwise it is undesired.

- Step 3: Prune all summaries with "desired" linguistic label. In our case, we used "OK" as "desired" linguistic label for all linguistic variables.

- Step 4: Order the summaries according to the priority. In our case this is according to the importance of the summarizer label.

- Step 5: Display summaries: maximally one for desired case and maximally two for undesired case.

\section{$5 \quad$ Results}

We will show now several examples of data with linguistic summaries obtained. Most of those examples are real data obtained from farms in the Netherlands from February 2018 to February 2019. A few dummy examples are shown to demonstrate extreme cases. We divided those examples in two groups: 
- type 1: undesired case

- type 2: desired case

\subsection{Type 1: Undesired Case}

In this subsection we show three examples that we classified as undesired case. We defined the case as undesired, when most of data is not in the desired range. The first example is depicted in Fig. 3 and shows daily range of temperature. Please note that desired values are on one extreme. Only a few data points can be described as ok. In this case two summaries are presented to the user:

- certain number (30\%-50\%) of differences in temperature during the day were too high $\left(6^{\circ} \mathrm{C}\right.$ or more $)$,

- certain number (30\%-50\%) of differences in temperature during the day were slightly high $\left(3-5{ }^{\circ} \mathrm{C}\right)$.

The first of them is about too high differences in temperature, since this linguistic label has the highest priority than the others, as indicated by the farmers.

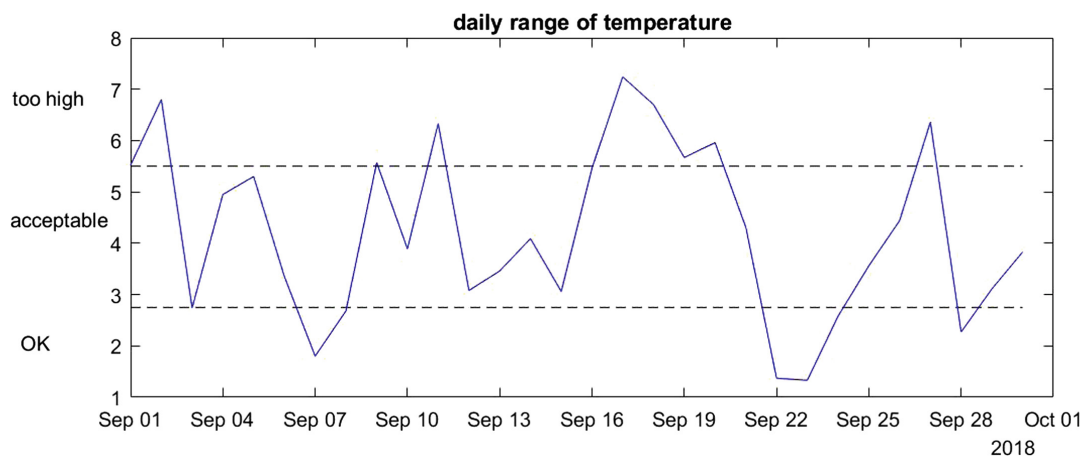

Fig. 3. Example: daily range of temperature - an undesired case

Another example (Fig. 4) depicts the levels of $\mathrm{CO}_{2}$ in a barn. Here again two summaries were obtained:

- Few (5\%-35\%) of the $\mathrm{CO}_{2}$ values were too high (3200 ppm or more),

- A lot (55\%-75\%) of the $\mathrm{CO}_{2}$ values were slightly high (2600-3200 ppm).

Even more that there are many more observations with only slightly high $\mathrm{CO}_{2}$ values, farmers indicated that the ordering should be done based on the importance of the summarizer, rather than qualifier.

The last example (Fig. 5) for this type of undesired cases is when only one summary is found relevant to be shown to the user.

- Most (80\%-95\%) of the humidity values were too low (below 40\%).

Please note, that this data are dummy data, created for the completeness purpose. 


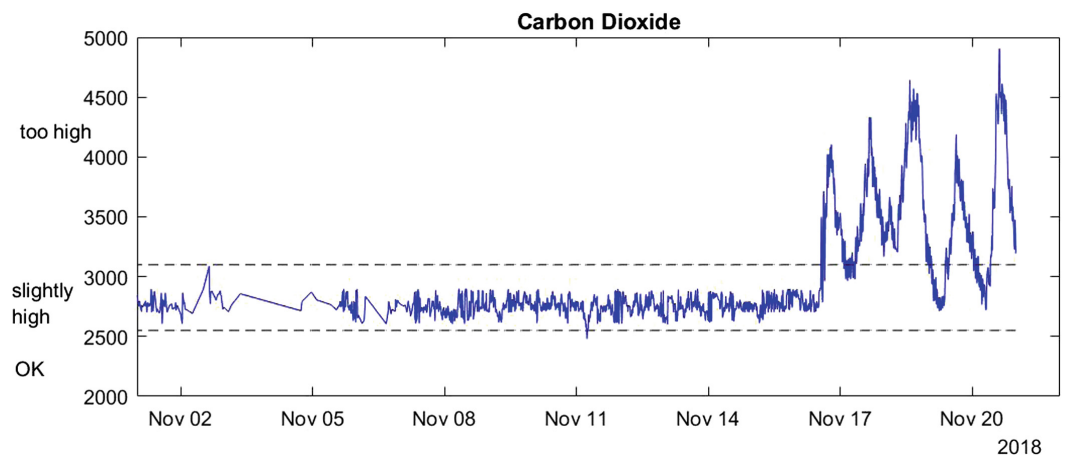

Fig. 4. Example: $\mathrm{CO}_{2}$ values - an undesired case

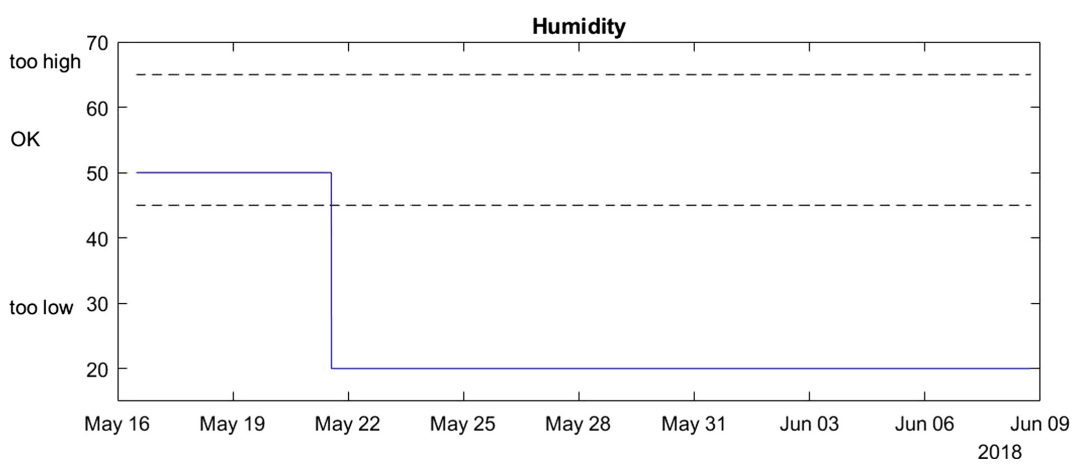

Fig. 5. Example: humidity values - an undesired case (dummy data)

\subsection{Type 2: Desired Case}

In this subsection we show two examples that we classified as desired case, that is when most of data is in the desired range.

The first example (Fig. 6) shows the shows daily range of temperature, with majority of values being ok.

Hence, in this case only a summary shown below is presented to the user.

- few (5\%-25\%) of differences in temperature during the day were slightly high $\left(3-5{ }^{\circ} \mathrm{C}\right)$.

The other example (Fig. 7) depicts $\mathrm{CO}_{2}$ levels. All the values were in the $O K$ range, hence in this case no summary is presented to the user.

\subsection{Evaluation with the Users}

We have evaluated this method with farmers. First the farmers were using the dashboard for two weeks, next they were asked to fill the questionnaire. Only 12 farmers has filled the questionnaire. The questionnaire was based on Technology 


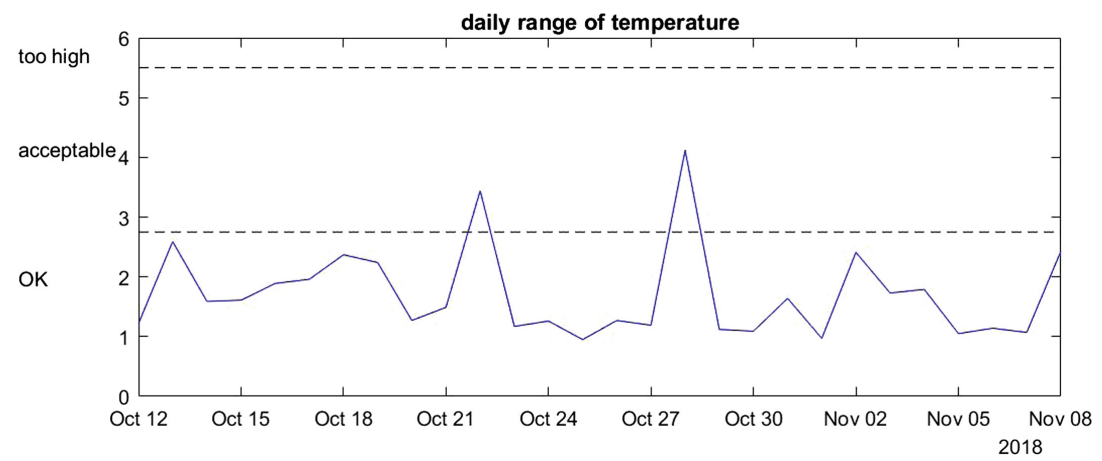

Fig. 6. Example: daily range of temperature - an desired case

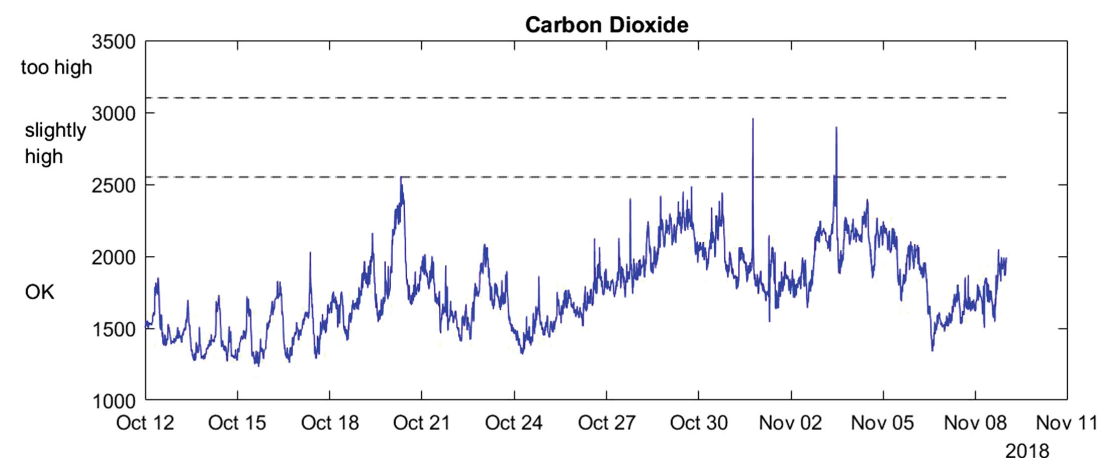

Fig. 7. Example: $\mathrm{CO}_{2}$ levels - an desired case

Acceptance Model (TAM) [25]. TAM assess four aspects: perceived ease of use, perceived usefulness, satisfaction and intention to use [26]. We have used the following 14 statements evaluated on the 7 point Likert scale:

Q1: Using LSs enable farmers to get information from the barn quickly.

Q2: Using LSs saves time of the farmers.

Q3: Using LSs improves my tracking efficiency of the barn.

Q4: Using LSs improves performance of the barn.

Q5: It is clear and understandable what are the LSs.

Q6: I find easy to read the LSs.

Q7: The words used in the LSs are easy and adequate.

Q8: I find good that the system calculates automatically the LSs.

Q9: I am completely satisfied with having sentences in the dashboard.

Q10: I am completely satisfied with the LSs that were presented.

Q11: I feel confident on using the LSs presented.

Q12: I can accomplish adjustments to barn quickly using LSs.

Q13: I will use LSs for tracking variables of the barn.

Q14: I would recommend to use LSs to other farmers. 

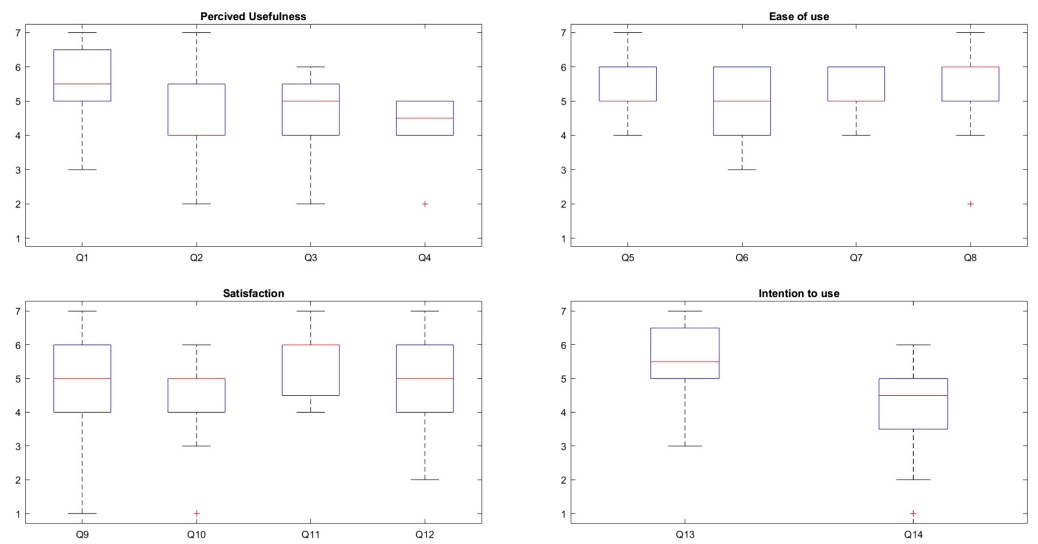

Fig. 8. Average scores for the results of TAM survey

The results of the survey are shown in Fig. 8.

The statements with the lowest score were "using LSs improves performance of the barn" from perceived usefulness aspect and "I would recommend to use LSs to other farmers" from intention to use. Analyzing the different scores of each farmer and the comments they did, it can be explained that these scores were low because according to some of them, it was expected that the linguistic summaries showed an alert instead of showing a summary of the past. Moreover farmers are reluctant to tell other farmers what to do.

On the other hand, the highest scores were for "using LSs enable farmers to get information about the barn quickly" from perceived usefulness; "I feel confident on using the LSs presented" from satisfaction; and "I will use LSs for tracking the variables of the barn" from intention to use aspect. This shows that, in general, farmers trust in the system and believe that linguistic messages are a good tool for making decisions in the barns.

When analyzing the factors in general, ease of use was the best characteristic of LSs, whereas perceived usefulness was the lowest score among the factors, but not with a significant difference. These results showed that linguistic messages are an appropriate solution for the farmers as they find it easy to use, but there is still more room for improvement on usefulness for the information provided.

To sum up, linguistic summaries are a good tool for the farmers; however, the linguistic texts that farmers expected were more about an alerting system, rather than a summarization system, which means that for the future, there is still some potential for extending the linguistic summaries with messages that not only describe the past, but support also decision making.

\section{Concluding Remarks}

We have analyzed and described an application of linguistic summaries in the agro-food domain. We have focused on the relevance aspect. We determine which 
linguistic summaries are useful and appropriate for target users through the interviews. The designed method was implemented and tested with the users. The results of a TAM survey indicated that linguistic summaries allow farmers to understand quickly the past performance, yet the farmers would appreciate also an alert functionality and more support in decision making. In future work we will extend the linguistic summaries with the suggestions of the users, as well as work on the additional case studies to generalize the approach. This should lead to a new quality measure quantifying relevance of a linguistic summary.

\section{References}

1. Anderson, D., Luke, R.H., Keller, J.M., Skubic, M., Rantz, M., Aud, M.: Linguistic summarization of video for fall detection using voxel person and fuzzy logic. Comput. Vis. Image Underst. 1(113), 80-89 (2009)

2. Anderson, D., Luke, R.H., Keller, J.M., Skubic, M., Rantz, M., Aud, M.: Modeling human activity from voxel person using fuzzy logic. IEEE Trans. Fuzzy Syst. 1(17), 39-49 (2009)

3. Anderson, D., Luke, R.H., Stone, E., Keller, J.M.: Segmentation and linguistic summarization of voxel environments using stereo vision and genetic algorithms. In: Proceedings IEEE International Conference on Fuzzy Systems, World Congress on Computational Intelligence, pp. 2756-2763 (2010)

4. Baczko, T., Kacprzyk, J., Zadrozny, S.: Towards knowledge driven individual integrated indicators of innovativeness. In: Knowledge-Based Intelligent System Advancements: Systemic and Cybernetic Approaches, pp. 129-140. IGI Global (2011)

5. Bugarín, A., Marín, N., Sánchez, D., Trivino, G.: Aspects of quality evaluation in linguistic descriptions of data. In: 2015 IEEE International Conference on Fuzzy Systems (FUZZ-IEEE), pp. 1-8 (2015)

6. Castillo-Ortega, R., Marín, N., Sánchez, D., Tettamanzi, A.G.B.: Quality assessment in linguistic summaries of data. In: Greco, S., Bouchon-Meunier, B., Coletti, G., Fedrizzi, M., Matarazzo, B., Yager, R.R. (eds.) IPMU 2012. CCIS, vol. 298, pp. 285-294. Springer, Heidelberg (2012). https://doi.org/10.1007/978-3-642-31715$6 \_31$

7. Castillo-Ortega, R., Marìn, N., Sànchez, D.: A fuzzy approach to the linguistic summarization of time series. Multiple-Valued Log. Soft Comput. 17(2-3), 157$182(2011)$

8. Delgado, M., Ruiz, M.D., Sanchez, D., Vila, M.A.: Fuzzy quantification: a state of the art. Fuzzy Sets Syst. 242, 1-30 (2014). https://doi.org/10.1016/j.fss.2013.10. 012

9. Díaz, C.A.D., Pérez, R.B., Morales, E.V.: Using linguistic data summarization in the study of creep data for the design of new steels. In: 2011 11th International Conference on Intelligent Systems Design and Applications, pp. 160-165. IEEE (2011)

10. Dijkman, R.M., Wilbik, A.: Linguistic summarization of event logs - a practical approach. Inf. Syst. 67, 114-125 (2017)

11. Few, S.: Information Dashboard Design: The Effective Visual Communication of Data. O'Reilly (2006)

12. Flach, P.: Machine Learning: The Art and Science of Algorithms That Make Sense of Data. Cambridge University Press, Cambridge (2012) 
13. Hastie, T., Tibshirani, R., Friedman, J.: The Elements of Statistical Learning: Data Mining, Inference, and Prediction. Springer, Heidelberg (2009)

14. Jain, A., Keller, J.M.: On the computation of semantically ordered truth values of linguistic protoform summaries. In: 2015 IEEE International Conference on Fuzzy Systems, FUZZ-IEEE 2015, Istanbul, Turkey, 2-5 August 2015, pp. 1-8 (2015)

15. James, G., Witten, D., Hastie, T., Tibshirani, R.: An Introduction to Statistical Learning, vol. 112. Springer, Heidelberg (2013). https://doi.org/10.1007/978-14614-7138-7

16. Kacprzyk, J.: Intelligent data analysis via linguistic data summaries: a fuzzy logic approach. In: Decker, R., Gaul, W. (eds.) Classification and Information Processing at the Turn of Millennium, pp. 153-161. Springer, Heidelberg (2000). https://doi. org/10.1007/978-3-642-57280-7_17

17. Kacprzyk, J., Strykowski, P.: Linguistic summaries of sales data at a computer retailer: a case study. In: Proceedings of IFSA 1999, vol. 1, pp. 29-33 (1999)

18. Kacprzyk, J., Wilbik, A.: Towards an efficient generation of linguistic summaries of time series using a degree of focus. In: Proceedings of the 28th North American Fuzzy Information Processing Society Annual Conference - NAFIPS 2009 (2009)

19. Kacprzyk, J., Wilbik, A., Zadrożny, S.: Linguistic summarization of time series using a fuzzy quantifier driven aggregation. Fuzzy Sets Syst. 159(12), 1485-1499 (2008)

20. Kacprzyk, J., Wilbik, A., Zadrożny, S.: An approach to the linguistic summarization of time series using a fuzzy quantifier driven aggregation. Int. J. Intell. Syst. 25(5), 411-439 (2010)

21. Kacprzyk, J., Yager, R.R.: Linguistic summaries of data using fuzzy logic. Int. J. Gen. Syst. 30, 33-154 (2001)

22. Kacprzyk, J., Yager, R.R., Zadrożny, S.: A fuzzy logic based approach to linguistic summaries of databases. Int. J. Appl. Math. Comput. Sci. 10, 813-834 (2000)

23. Kacprzyk, J., Zadrożny, S.: Fuzzy linguistic data summaries as a human consistent, user adaptable solution to data mining. In: Gabrys, B., Leiviska, K., Strackeljan, J. (eds.) Do Smart Adaptive Systems Exist?, vol. 173, pp. 321-339. Springer, Heidelberg (2005). https://doi.org/10.1007/3-540-32374-0_16

24. Kacprzyk, J., Zadrożny, S.: Linguistic database summaries and their protoforms: toward natural language based knowledge discovery tools. Inf. Sci. 173, 281-304 (2005)

25. Kwon, H.S., Chidambaram, L.: A test of the technology acceptance model: The case of cellular telephone adoption. In: Proceedings of the 33rd Annual Hawaii International Conference on System Sciences, pp. 7-pp. IEEE (2000)

26. Lai, P.: The literature review of technology adoption models and theories for the novelty technology. JISTEM-J. Inf. Syst. Technol. Manag. 14(1), 21-38 (2017)

27. Lewis, R.L.: Interference in short-term memory: the magical number two (or three) in sentence processing. J. Psycholinguist. Res. 25(1), 93-115 (1996)

28. Marín, N., Sánchez, D.: On generating linguistic descriptions of time series. Fuzzy Sets Syst. 285, 6-30 (2016). https://doi.org/10.1016/j.fss.2015.04.014. Special Issue on Linguistic Description of Time Series

29. Mkhabela, M., Bullock, P., Raj, S., Wang, S., Yang, Y.: Crop yield forecasting on the Canadian prairies using MODIS NDVI data. Agric. Forest Meteorol. 151(3), 385-393 (2011)

30. Moyse, G., Lesot, M.J., Bouchon-Meunier, B.: Linguistic summaries for periodicity detection based on mathematical morphology. In: 2013 IEEE Symposium on Foundations of Computational Intelligence (FOCI), pp. 106-113 (2013) 
31. Panda, S.S., Ames, D.P., Panigrahi, S.: Application of vegetation indices for agricultural crop yield prediction using neural network techniques. Remote Sens. 2(3), 673-696 (2010)

32. Pretorius, A.J., Van Wijk, J.J.: What does the user want to see? What do the data want to be? Inf. Vis. 8(3), 153-166 (2009)

33. Reiter, E.: Non-experts struggle with information graphics (2017). https:// ehudreiter.com/2017/10/02/non-experts-struggle-graphs/

34. Rong, A., Akkerman, R., Grunow, M.: An optimization approach for managing fresh food quality throughout the supply chain. Int. J. Prod. Econ. 131(1), 421429 (2011)

35. Ros, M., et al.: Linguistic summarization of long-term trends for understanding change in human behavior. Proceedings of the IEEE International Conference on Fuzzy Systems, FUZZ-IEEE 2011, pp. 2080-2087 (2011)

36. Smits, G., Nerzic, P., Pivert, O., Lesot, M.: Efficient generation of reliable estimated linguistic summaries. In: 2018 IEEE International Conference on Fuzzy Systems, FUZZ-IEEE 2018, Rio de Janeiro, Brazil, 8-13 July 2018. pp. 1-8 (2018)

37. Szczepaniak, P.S., Ochelska, J.: Linguistic summaries of standardized documents. In: Last, M., Szczepaniak, P.S., Volkovich, Z., Kandel, A. (eds.) Advances in Web Intelligence and Data Mining, vol. 23, pp. 221-232. Springer, Heidelberg (2006). https://doi.org/10.1007/3-540-33880-2_23

38. Wilbik, A., Dijkman, R.: Linguistic summaries of process data. In: Proceedings of IEEE International Conference on Fuzzy Systems (FUZZ-IEEE 2015) (2015)

39. Wilbik, A., Keller, J.M., Alexander, G.L.: Linguistic summarization of sensor data for eldercare. In: Proceedings of the IEEE International Conference on Systems, Man, and Cybernetics (SMC 2011), pp. 2595-2599 (2011)

40. Wong, P.C., Thomas, J.: Visual analytics. IEEE Comput. Graph. Appl. 5, 20-21 (2004)

41. Yager, R.R.: A new approach to the summarization of data. Inf. Sci. 28, 69-86 (1982) 